Research Article

\title{
Experience Control Analysis of English Reading Software Based on Wireless Binocular Line-of-Sight Sensing
}

\author{
Cheng Feng $\mathbb{B}$ \\ Department of Foreign Languages, Nanchong Vocational and Technical College, Nanchong, 637000 Sichuan, China \\ Correspondence should be addressed to Cheng Feng; 1812230030@e.gzhu.edu.cn
}

Received 14 September 2021; Revised 14 October 2021; Accepted 16 October 2021; Published 28 October 2021

Academic Editor: Guolong Shi

Copyright (C) 2021 Cheng Feng. This is an open access article distributed under the Creative Commons Attribution License, which permits unrestricted use, distribution, and reproduction in any medium, provided the original work is properly cited.

\begin{abstract}
This paper proposes a segmented combined English text measurement method based on two sets of orthogonal linear image sensors and one area image sensor. This method fully combines the advantages of the linear image sensor and the area image sensor in longdistance and short-distance English text measurement and can continuously perform high-precision English text tracking within a large range of viewing distance. Based on this method, a set of segmented English text measurement system is designed and constructed. This paper presents a method for extracting English word boundaries based on semantic segmentation to solve the problem of global positioning and horizontal initialization of English reading text. The semantic segmentation method based on fully convolutional networks (FCN) is analyzed, and the target classification is defined. We used the classic FCN framework and model, fine-tuned with manually annotated data, and achieved good segmentation results. For the definition and extraction of English word boundaries in English text, a piecewise linear model is used to measure the projection confidence of each English word boundary point, and the overall observation of the English word boundary is measured. When the observation confidence is high enough, combined with the English word boundaries marked in the high-precision image, the horizontal positioning is obtained by matching the weights. This paper concludes that English reading software can help learners in English learning to a certain extent, which proves that the English reading software is an effective supplement based on blended learning classrooms. Through the analysis of learners and teaching content, an English teaching model based on English reading software blended learning is designed. Experimental studies have proved that English reading software can help learners learn English, which not only expands their vocabulary but also broadens their horizons.
\end{abstract}

\section{Introduction}

The camera is a type of computer peripheral that is currently widely used. It uses an ordinary lens and a CMOS/CCD sensor to obtain an image of the subject. After special DSP processing and encoding into MPEG or other standard audio and video data, using some network interactive software, people can share each other's image resources on this machine or with interactive communicators on the Internet $[1,2]$. However, the existing cameras use a single lens and a CMOS/CCD sensor to form a system, which can only obtain two-dimensional images of the subject without depth information, and the communicator cannot see the subject with stereo vision, which is difficult. The comprehensive acquisition of the three-dimensional data information of the subject makes the communication lack of real experience, and data loss is serious after the digital pro- cessing and restoration of the real environment [3]. In recent years, more and more people have devoted themselves to the research work of computer stereo vision technology [4]. How to obtain the image of the photographed object is the basis of the entire stereo vision image acquisition system. 3D vision images generally use binocular images that simulate human eyes, but there are also many multieye images that use more than two cameras. The acquisition of binocular images is usually done by two cameras with a certain displacement on the same horizontal line.

English is the common language of the world today. Most countries in the world use English in diplomatic activities, government documents, official meetings, etc.; $72 \%$ of emails are done in English; 70\% of publications in the world are written in English [5]. It seems that English has become world's most common language. As a compulsory course 
from basic education to higher education in our country, English plays an important role in people's study, work, and life. With the popularization of mobile devices and the improvement of their functions, various mobile learning platforms are gradually being promoted, and more and more people use mobile devices for mobile learning [6]. Mobile technology provides good support for text, pictures, audio, video, animation, files, and other elements. It is quickly applied to the field of English learning. Many Englishrelated mobile learning platforms have begun to appear, such as handheld listening, speaking, and sailing English learning. The English course advocates the English teaching concept of quality education, provides healthy and active teaching materials, and lays a good English foundation for students. It highlights the main status of learners and gives full play to the guiding role of teachers [7]. Good use of modern educational technology and vigorously encouraging learners to learn English through remote or online platforms can improve efficiency.

In order to solve the existing problem of large viewing distance relative to English text measurement in visual navigation applications, this paper proposes a segmented combined English text measurement method and gives a system implementation plan. Specifically, the technical contributions of this article can be summarized as follows:

First, we divide the measurement task into the far segment and the near segment according to the difference of the measurement distance. The corresponding system includes a far-segment measurement module composed of two sets of orthogonal linear image sensors, a near-segment measurement module composed of an area array image sensor, and a cooperative target light source composed of near-infrared LEDs. This paper proposes a method for global positioning and initialization of English reading text based on semantic segmentation. The word-level horizontal positioning is obtained, which provides a horizontal initial value for the global positioning of subsequent English reading text.

Second, this article matches English word boundaries with images. We propose a pixel-level semantic segmentation method based on deep learning network. A semantic segmentation map is generated, and then, dynamic and static targets are analyzed to obtain English word boundaries.

Third, according to the plane assumption, the English word boundary in the image coordinate system is projected to the coordinate system, and it is matched with the English word boundary in the high-precision image to obtain the word-level positioning result. At the same time, the surrounding dynamic target interference is evaluated, which improves the robustness to dynamic target interference.

Fourth, it is proved from a quantitative perspective that English reading software has a positive impact on the improvement of learners' language proficiency. The experimental results show that the use of English reading software in the teaching of blended English courses can help learners improve their English reading performance. Based on the analysis of the questionnaire survey data and interviews, this article concludes that learners agree with English reading software. It can be seen that the English reading software environment is a further extension of classroom learning.
It not only changes the teaching mode of teachers but also makes great changes to the learning mode of students.

\section{Related Work}

Related scholars use Web technology to develop an adaptive English learning system for college students to promote the independent and personalized learning of English learners [8]. Researchers develop an English learning system with students as the main body, providing learning materials, learning tools, learning evaluation, interactive communication, and other functional modules [9]. Learners can choose relevant learning materials for learning based on their own knowledge. Teachers can enter the background to view students' learning situation and sharing of teaching results. Relevant scholars have developed a web-based English teaching platform based on the students of higher vocational colleges [10]. The platform provides functions such as grade exams, teaching supplementary topics, online self-tests, special topics, and interactive platforms to improve students' interest in English learning. Related scholars develop a mobile listening learning system for specific users with college students, providing functions such as vocabulary, reading, and testing, and improve students' listening skills through effective training for students [11]. It takes college students as the research object, with vocabulary learning as the main content, and develops a mobile English learning platform based on the Android system in order to create a good learning environment and improve the learning efficiency and learning efficiency of English learners.

Research on basic English learning through the ELearning system has become a hot issue for foreign ELearning scholars [12]. Scholars at the School of Information, Kyoto University in Japan use the E-Learning system to intelligently judge whether the pronunciation of English learners in elementary and middle schools is correct or not. Scholars from the Language Learning and Nature Laboratory of Simon Fraser University in Canada use the E-Learning system to enable elementary and middle school students to get timely feedback when learning English [13]. The feedback given by the system includes word spelling check, grammar check, and pronunciation check. In addition to the legacy word check, two scholars conducted a survey of learners who used this system, and 79\% of the students were satisfied with the effect of the system [14]. The scholars from Ho Chi Minh City University of Science and Technology in Vietnam jointly used the E-Learning system to conduct related research on the writing evaluation of English learners in junior high schools [15]. The three scholars used an algorithm to use computers to help teachers evaluate students' compositions, reducing teachers' work pressure and using experiments to prove that the accuracy of the evaluation results using this system reached 87\%, which improved the English teachers' performance in junior middle schools [16].

The scholars of the Autonomous University of Barcelona, Spain, through the online learning environment created by the E-Learning system, select 78 primary school students from Spanish primary schools and British primary schools for one-on-one mutual aid language learning [17]. Scholars have proved through experiments that with the help of the online 
learning environment, the language learning level of mutual aid learners has been greatly improved [18]. Relevant scholars select abstract words in the English learning stage of primary and secondary schools as the content of E-Learning, conduct network learning through the E-Learning system, and conduct comparative experimental results [19]. It is proved that primary school students who learn abstract English words through the E-Learning system have better academic performance. Students who learn through classroom explanations are high. It can be seen that by designing an E-Learning English learning system that meets the needs of students and teachers, English learners can improve their English listening, reading, oral dialogue, and writing skills, as well as help English teachers improve their teaching ability and teaching philosophy [20].

Relevant scholars have used Internet technology to build a hybrid education and teaching platform based on cloud computing technology under the cloud computing platform by analyzing the current low level of informatization in the education industry and the chaotic management of teaching resources [21]. The platform effectively integrates education and teaching resources, promotes the process of education informatization, and improves the effect of education and teaching. Relevant scholars have put forward a hybrid learning and teaching management platform that combines a network teaching management system and a teaching resource platform by analyzing the current development of Internet technology and the education industry [22]. This optimizes the existing teaching methods and improves the efficiency of school teaching resource management. Researchers analyzed the investigation of factors affecting learning satisfaction in a blended learning environment and found that students' learning atmosphere, learning motivation, and interactive behavior are three of the factors that affect students' learning satisfaction [23]. By designing and implementing a hybrid learning hierarchy model based on learning satisfaction, it effectively improves the learning effect of students and provides reference guidance for subsequent learning satisfaction model research.

\section{Segmented Visual English Text Measurement Design}

3.1. Overall Technical Solution. In order to meet the requirements of resolution, distance range, measurement speed, and reliability in actual measurement, this paper proposes a segmented combined English text measurement method and system implementation plan. This method divides the measurement task into a far segment and a near segment. Two sets of orthogonal linear image sensors form a far segment measurement module, and an area array image sensor forms a near segment measurement module. The near-infrared LED light source is used as the active sign on the target platform. The light source adopts a layered design method. It consists of an outer ring and an inner ring. The outer ring light source is composed of 4 high-power LEDs. Two medium-power LEDs form the inner ring light source to provide a cooperation sign for the near-segment measurement module. The system composition is shown in Figure 1.
When the distance between the English text measurement system and the target platform is less than 5 meters, the target exceeds the measurement field of view of the farsegment orthogonal linear image measurement module and enters the "near-segment" measurement range. In this range, the measurement accuracy of English text is required to be high. At this time, the near-segment monocular image measurement module is used to accurately measure target's English text, and the inner ring light source composed of 4 medium-power LEDs is used to provide a cooperation sign for the measurement module.

\subsection{The Design of Remote Orthogonal Linear Array Image} Measurement. In order to accurately control the image acquisition time of the four linear CCD cameras in the two camera groups in dynamic coordinate measurement, and to process the acquired images in parallel, a special image acquisition and processing circuit is designed for each linear CCD camera. The design of the circuit requires the ability to collect and process linear CCD signals in real time and to detect and output the pixel coordinates of the LED light source. This circuit is composed of three parts: linear CCD drive circuit, image buffer circuit, and image processing circuit.

The linear array CCD drive circuit mainly includes the linear array CCD image sensor, CPLD, amplifying circuit, $\mathrm{ADC}$, and tri-state buffer chip. The main function of this module is to provide the required drive signal for the normal operation of the linear array CCD, to amplify and convert the image signal output by the linear array CCD, and to provide the sequence needed for the subsequent image buffering.

The linear CCD image sensor is the core component of the vision measurement system. Its selection needs to consider key characteristic parameters such as sensitivity, dynamic range, resolution, frame rate, and spectral response. According to the task requirements of the English text measurement system in this subject, the TCD142D CCD chip from Toshiba was selected, with a resolution of $2048 \times 1$ pixels, a pixel size of $14 \mu \mathrm{m} \times 14 \mu \mathrm{m}$, a data rate of up to $10 \mathrm{MHz}$, and a good spectral response near the wavelength of $740 \mathrm{~nm}$. It has the characteristics of high sensitivity and low dark current, which can meet the needs of the system.

\subsection{Circuit Design of Text Image Processing in English Reading} Software. The workflow of the text image acquisition process of English reading software is shown in Figure 2. When the DSP receives the interrupt signal from the communication link, it immediately starts the FPGA to collect the image through the IO level. Since the communication link triggers synchronous acquisition by interruption, the image acquisition is a timely response. When the remote measurement module is working and the LED light source adopts stroboscopic modulation, the modulation frequency is set to $1 / 5$ of the frame rate, so 6 frames of images constitute a stroboscopic cycle. The DSP processes 6 frames of images as a unit, and the position of the target light source on the image can be obtained through background difference and demodulation. In the process of image access, the idea of ping-pong operation is adopted. FPGA writes images to two RAM memory blocks in a loop, and DSP reads images from free memory blocks. 


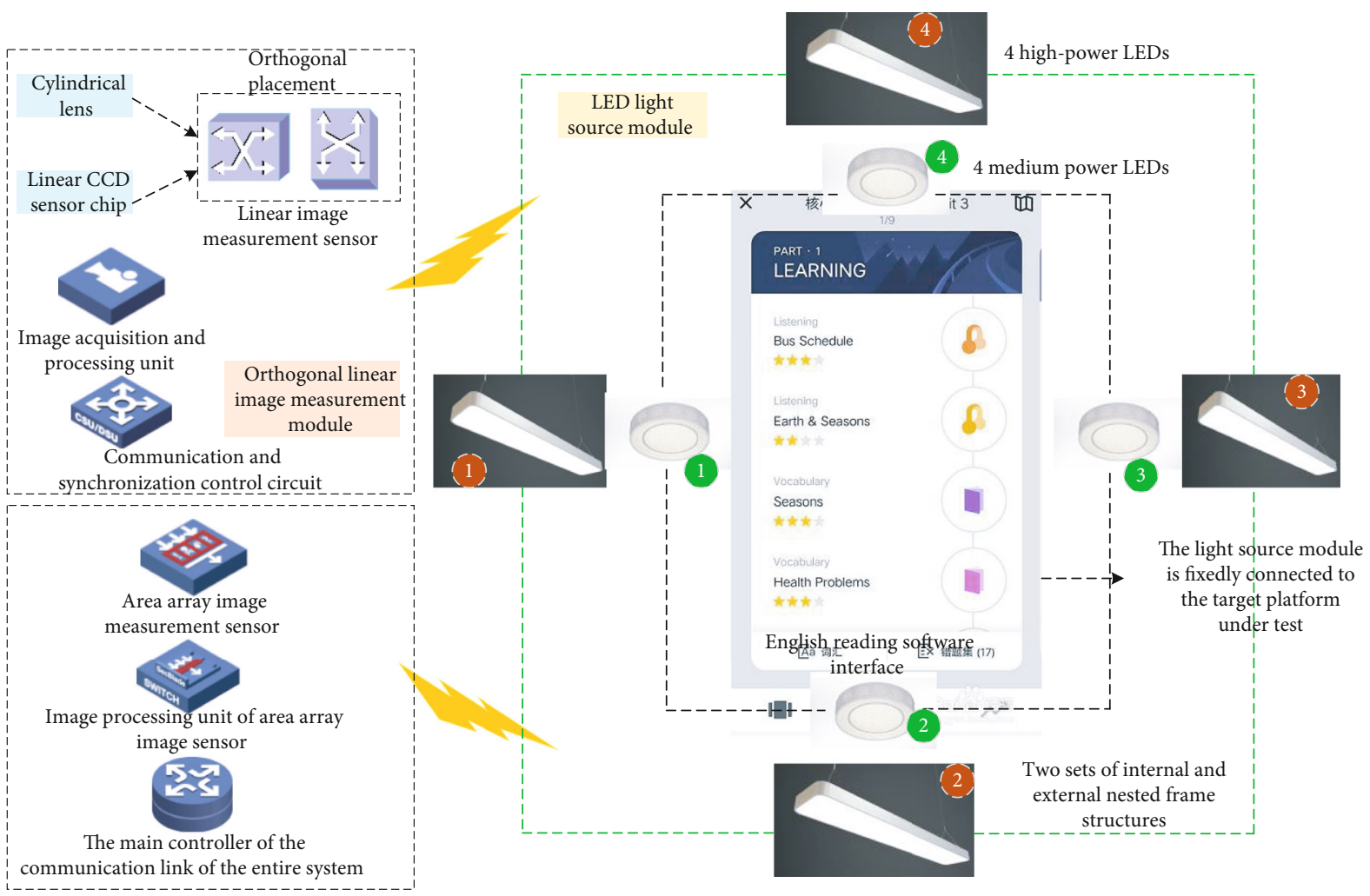

Figure 1: The overall design scheme of the English text measurement system.

The time interval of the synchronous trigger interrupt is a fixed value, which is greater than the time required for the FPGA to acquire 6 frames of images, so it can be guaranteed that the FPGA will write the current memory block before the trigger arrives.

\section{Global Positioning Algorithm for English Reading Text Based on Semantic Segmentation}

4.1. FCN Network. Traditional semantic segmentation mainly relies on manual feature extraction and pixel prediction and classification based on probability distribution. The classic method is to extract features through a deep convolutional neural network, layer by layer convolution pooling, and finally, complete tasks such as classification through a fully connected layer. A classic convolutional neural network structure contains a series of convolutional layers, pooling layers, and fully connected layers. Among them, the convolution layer uses multiple convolution kernels with different parameters to convolve local images to obtain multidimensional local features; the pooling layer reduces the dimensions of the multidimensional local features obtained by convolution in the spatial dimension, reducing the amount of calculation. The fully connected layer integrates a series of local features obtained above and outputs the probability of the image category according to the task target.
But in the traditional network structure, such as AlexNet and VGG-Net, the last few layers are basically fully connected layers, and the output is the result of target classification, and the pixel segmentation cannot be completed. full convolutional network (FCN) replaces the fully connected layer of the classification network with a convolutional layer and then upsamples the deconvolutional layer to obtain a prediction for each pixel category. In addition, the coarse high-level information and the fine low-level information were merged to obtain better segmentation results. A schematic diagram of semantic segmentation based on a fully convolutional neural network is shown in Figure 3.

\subsection{Establishment of Surround View Camera Dataset and} Network Training. The fisheye camera used in this article requires fine tune, which requires a lot of data. However, there is almost no database for semantic segmentation of fisheye camera images. In order to expand the dataset, this article uses the above-mentioned surround-view fisheye camera to collect fisheye images, including four fisheye cameras.

Deep learning network training needs to rely on a large number of training datasets, and this article only has a small-scale dataset. In order to prevent overfitting during the network training process, this paper adopts the transfer learning method for training. This article uses the pretrained model of FCN on the Cityscapes dataset to fine-tune. Since our dataset is small and there is a certain difference between the images in the Cityscapes dataset, this article will freeze the weights of 


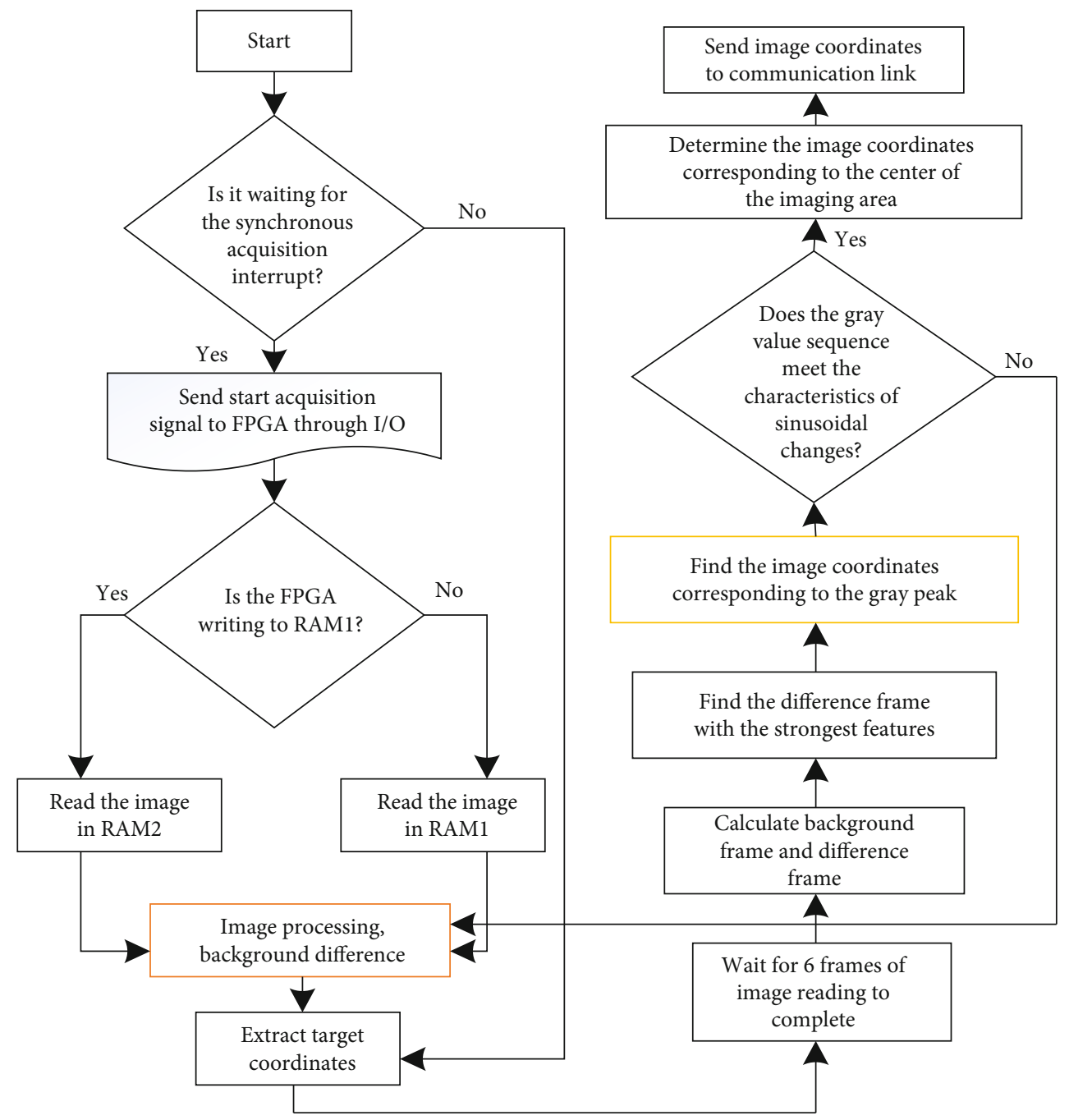

FIGURE 2: The workflow of the text image collection process of English reading software.

the previous convolutional layers in the pretraining model and only retrain the last two convolutional layers.

4.3. English Word Boundary Detection and Extraction. In English text, English word boundaries are difficult to clearly define, and rule-based detection of English word boundaries is extremely difficult. This article divides English word boundaries into three major categories: F, S, and D.

The roadside boundary refers to the boundary of the $\mathrm{F}$ area, but the boundary between $\mathrm{D}$ and $\mathrm{F}$ is not a real English word boundary. Therefore, the English word boundary B in this article is defined as the boundary between $\mathrm{F}$ and $\mathrm{S}$ :

$$
B=\left[\left(b_{i}, I_{i}, E_{i+1}\right), \quad i=1,2, \cdots, N\right],
$$

where $N$ is the number of boundary points in the F area, $I_{i}$ represents the four fisheye camera images of the front, rear, left, and right; $b_{i}$ refers to the coordinates of the pixel in the image coordinate system, and $E_{i}$ represents whether it is a real English word boundary.

We use a series of appropriate line segments to mark the English word boundaries defined in this article, and discretize them at equal intervals as the English word boundary images. This paper uses high-precision images to mark the prior English word boundary images. The high-precision images used in this paper are all established by the image acquisition equipment of the intelligent laboratory. During the mapping process, the vehicle-mounted panoramic camera Ladybug-5 was used for image collection, and the high-precision GPS was used to obtain location information. The collected panoramic images are subjected to inverse perspective transformation and spliced to obtain a wide range of road images.

The process of constructing feature images can be abstracted as the process of finding the union of visual feature points in the navigation coordinate system.

$$
\operatorname{Map}=F_{0} \cup F_{1} \cup F_{2} \cup \cdots \cup F_{T-1} \cup F_{T} .
$$

$F_{T}$ represents the feature point set in the navigation coordinate system at time $T$.

4.4. Image Matching Based on Weighted ICP. Based on the semantic segmentation of English word boundary detection and the generation of English word boundary images, we 


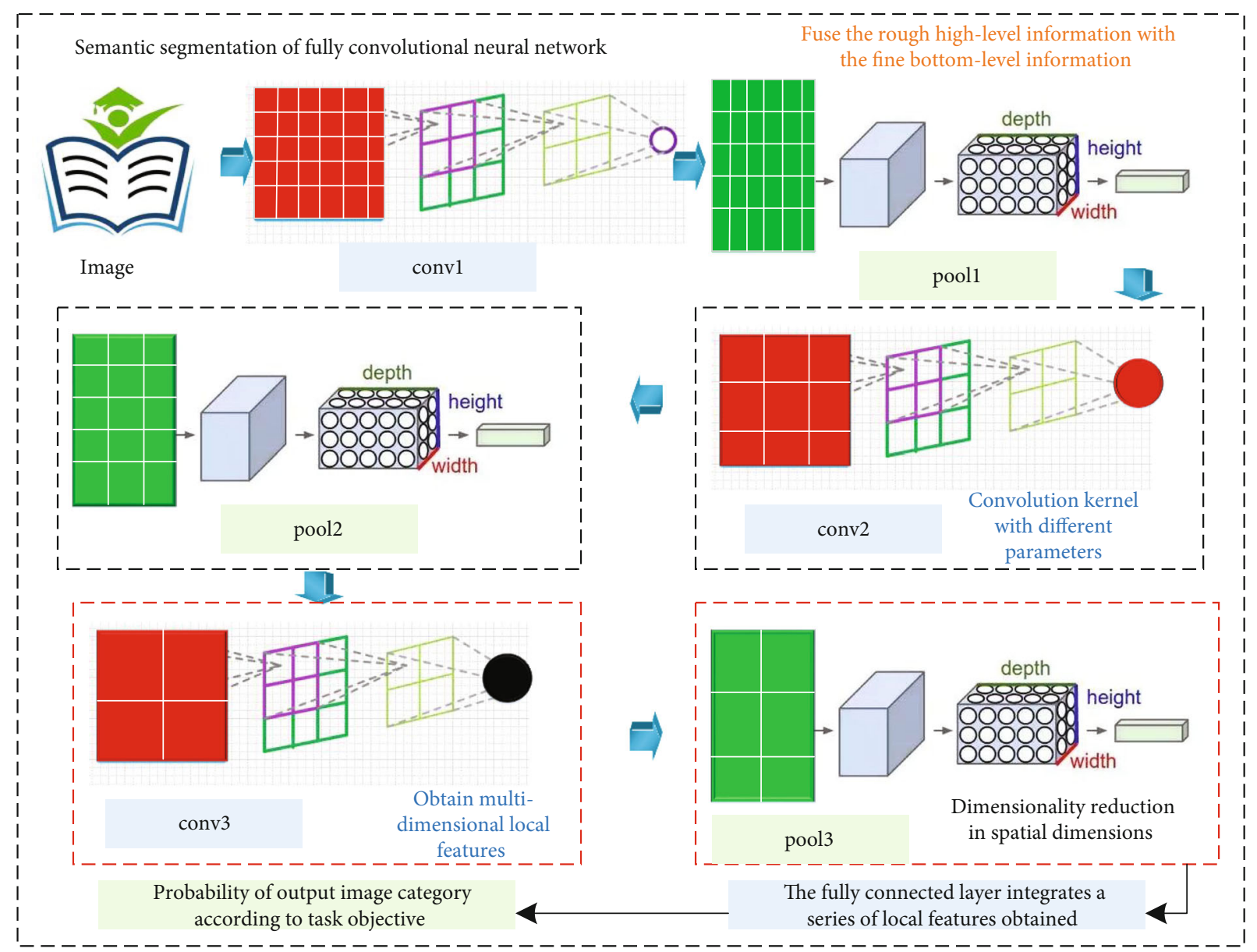

FIgURE 3: Schematic diagram of semantic segmentation based on fully convolutional neural network.

use the ICP algorithm to match the two to obtain positioning. The classic ICP algorithm is an iterative optimization process, constantly looking for corresponding points and then optimizing the Euclidean distance error between the corresponding points. However, there is a systematic error in the observation of the English word boundary through the inverse perspective transformation, that is, the farther the distance from the camera is, the greater the position error obtained by the projection; the closer the point, the higher the projection accuracy, so the classic ICP algorithm is required. To make improvements, the confidence of each observation point is introduced into the calculation of the distance error, which is called the weight ICP. The following formula gives the mathematical model of the error function in the weighted ICP:

$$
E(R,-T)=\operatorname{Min} \prod_{i=0}^{N-1} m_{i+1}\left(T+R p_{i}-q_{i}\right)^{2}
$$

Among them, $q_{i}$ and $p_{i}$ are the corresponding points, $m_{i}$ is the confidence of the corresponding point, and $R$ and $T$ are the rotation and translation matrices obtained through optimization. According to the previous analysis, the confidence is related to the distance between the projection point and the camera. The farther the distance, the lower the confidence, and the closer the distance, the higher the confidence. This paper defines a confidence evaluation model. When the distance between the projection point and the camera is less than the threshold Min, the confidence is 1; when the distance between the projection point and the camera is less than the threshold Min, the confidence is linearly reduced; when the distance is greater than Max, the confidence is 0 .

$$
w_{i}= \begin{cases}-1, & d_{i}>\operatorname{Max}, \\ \left(d_{i}-\operatorname{Min}\right) /(\operatorname{Max}+\operatorname{Min}), & \operatorname{Min} \leq d_{i} \leq \operatorname{Max}, \\ 1, & d_{i}<\operatorname{Min} .\end{cases}
$$

Although the weight ICP can obtain more accurate matching results in most scenes, in busy scenes, there are usually a large number of obstructions surrounding camera's line of sight, and it is impossible to detect enough English word boundary points. At this time, it is difficult to match. Or the English word boundary points are far away, and the projection error will increase, the observation confidence will decrease, and the matching result will not be very good. 
TABLE 1: Analysis of usual results.

\begin{tabular}{lccccc}
\hline Class & Number of people & Average value & Standard deviation & $T$ value & Significance \\
\hline Experimental class & 50 & 87.5 & 2.4 & 1.26 & 0.179 \\
Control class & 50 & 86.8 & 2.1 & 1.26 & 0.179 \\
\hline
\end{tabular}

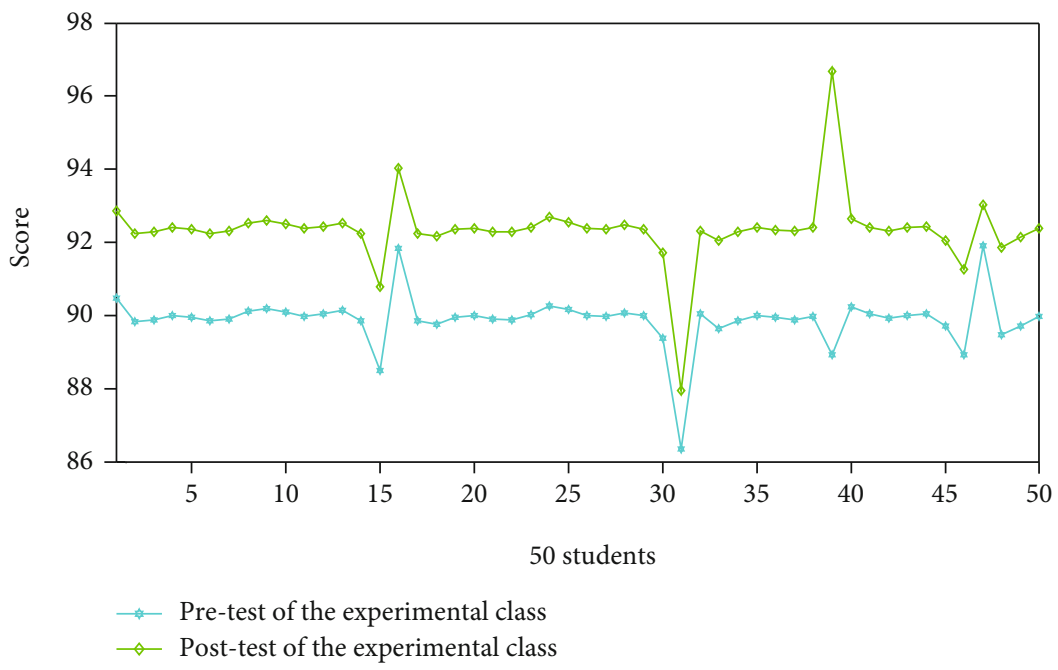

Figure 4: Comparison of test results before and after the experimental class.

Therefore, it is necessary to consider whether matching can be performed before matching. The above formula gives the confidence of a single projection point. In this paper, the average confidence of all projection points observed in each frame is used as a priori evaluation. Only the average confidence is higher than the threshold for matching positioning.

$$
\begin{gathered}
w=\prod_{i=1}^{N-1} \frac{w_{i}}{(N-1)}, \\
V_{k}=\left(w \longrightarrow w^{\prime} \leq w_{t h r}\right) .
\end{gathered}
$$

\section{Experiment and Analysis}

5.1. Analysis of Observation Records. This article sorts out the usual performance scores of the experimental class and the control class during the experiment. Normal performance scores consist of classroom performance scores and homework scores, of which class performance scores and homework scores both account for 50\%. This article uses SPSS22.0 to make a statistical analysis of the results of the two classes. The analysis results are shown in Table 1.

It can be seen from Table 1 that the average scores of the experimental class and the control class are 87.5 and 86.8, respectively, the $T$ value is 1.26 , and the two-tailed test significance $P$ value is 0.179 . Since $P>0.05$, it shows that there is no obvious difference between the average scores of the experimental class and the control class. It can be explained that even though the average value of the experimental class's usual scores is slightly greater than the average of the control class's usual scores, there is no significant difference between the two. In other words, the effect of the English reading APP on students' performance in the English classroom and homework during the experiment was not particularly obvious. This may be related to students' learning motivation, self-learning ability, and self-discipline.

5.2. Analysis of Test Results. Before the experiment, the instructor tested the experimental class and the control class, respectively. The average pretest score of the experimental class was slightly higher than the average test score of the control class. The $F$ value of the test for the homogeneity of variance of the previous test scores did not reach the significant level $(P>0.05)$, and the hypothesis of homogeneity of variance was accepted, that is, the variances of the two classes are equal. The $T$ value is 1.42 , and the two-tailed test significance $P$ value is 0.152 . Since $P>0.05$, there is no significant difference between the experimental class and the control class. After the experiment, the instructor tested the experimental class and the control class. The comparison of the test scores before and after the experimental class is shown in Figure 4, and the comparison of the test results before and after the control class is shown in Figure 5.

Comparing the scores of the experimental class and the control class before and after the test, it can be seen that learners' reading test scores have improved. This article believes that the reason for this change is that both the experimental class and the control class are learning English during the experiment. After the experiment, both the experimental class and the control class improved. However, the $T$ value of the average posttest score of the experimental 


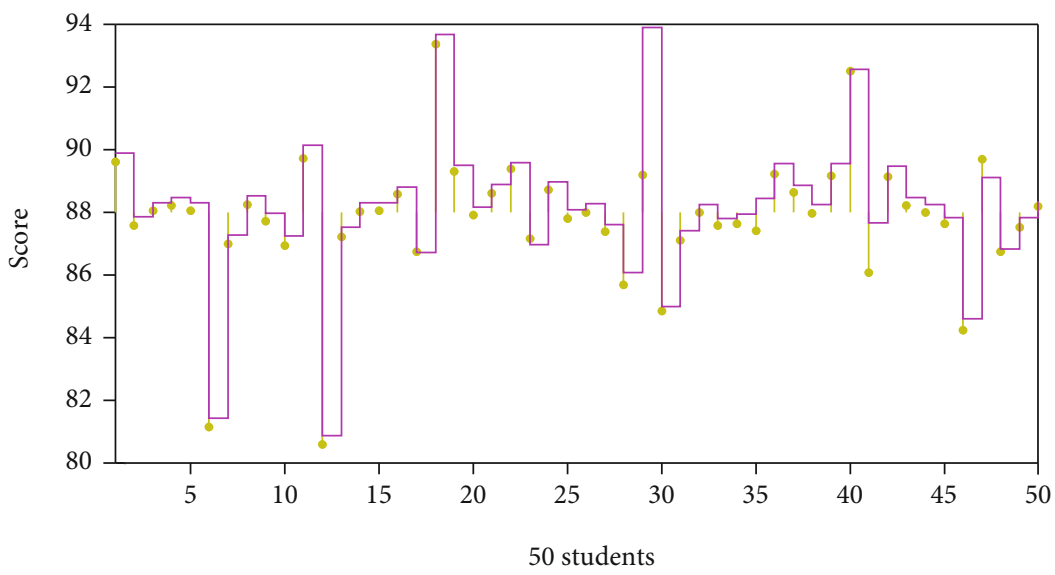

$\longrightarrow$ Pre-test of the experimental class

_ Post-test of the experimental class

FIgURE 5: Comparison of test scores before and after the control class.

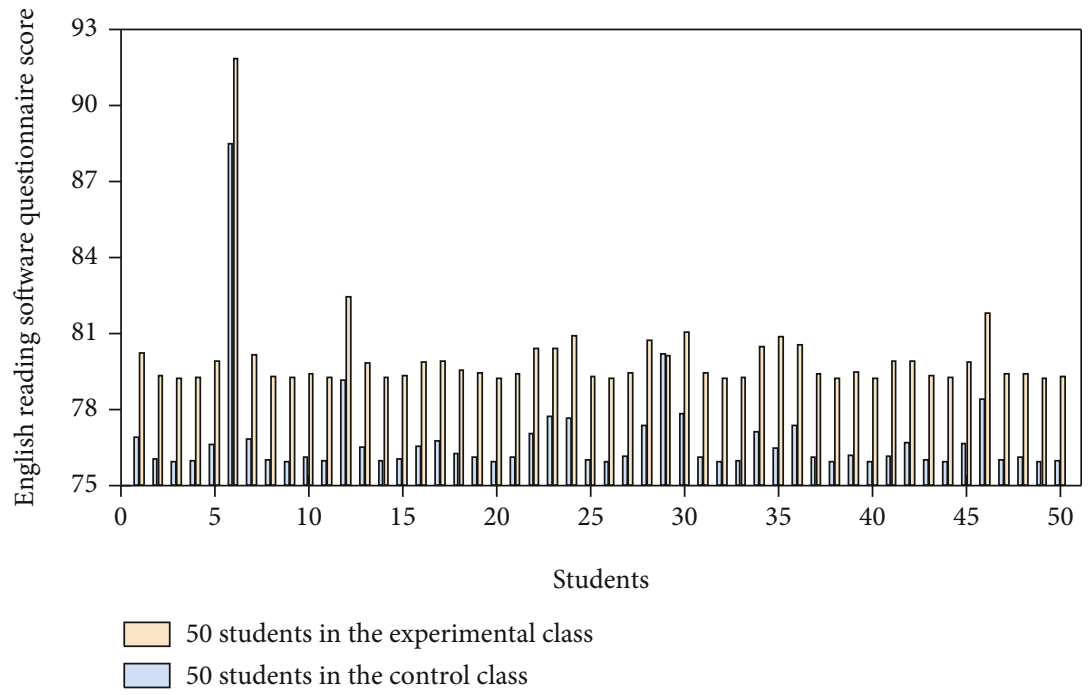

FIgURE 6: Histogram of questionnaire score analysis.

class and the control class is 2.927 , and the two-tailed test $P$ value is $0.004(P<0.05)$. There are significant differences in scores. In other words, the performance of the experimental class improved more than that of the control class. This shows that through the use of English reading software for English reading learning, learners' English reading test scores have been improved to a certain extent.

5.3. Analysis of the Results of the Questionnaire Survey. There are a total of 21 questions in this questionnaire. The first 20 questions are closed-ended questions, and the 21st question is an open-ended question. The answers to the closed questions take the form of a Likert 5-point scale, where 1 represents "strongly disagree," 2 represents "disagree," 3 represents "uncertain," 4 represents "agree," and 5 represents "very agree." The questionnaire has a total score of 100 points. The questionnaire has 20 Likert scale questions. The total score is 20 points for very disagree, 40 points for disagreement, 60
TABle 2: Approval degree of knowledge and skills dimension.

\begin{tabular}{lcc}
\hline $\begin{array}{l}\text { First-level } \\
\text { dimension }\end{array}$ & Secondary dimension & $\begin{array}{c}\text { Agree } \\
(\%)\end{array}$ \\
\hline & $\begin{array}{c}\text { Article information analysis } \\
\text { Cultural background }\end{array}$ & 59 \\
Knowledge and & knowledge & 77 \\
skills & Statement & 64 \\
& Vocabulary & 69 \\
\hline
\end{tabular}

points for uncertainty, 80 points for agree. The total score of the students in the experimental class represents their evaluation of the use of the English reading software. The higher the score, the more the experimental subjects agree with the use of the English reading software, and vice versa.

After the experiment, this paper carried out the on-site distribution and recovery of questionnaires. 100 questionnaires 


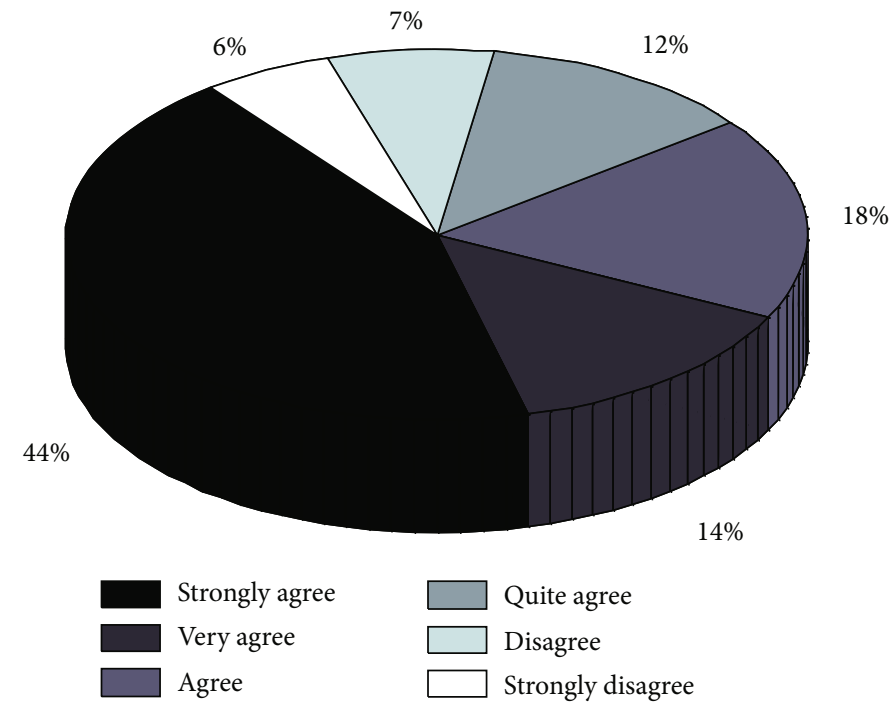

Figure 7: Proportion of approval degree of process and method dimensions.

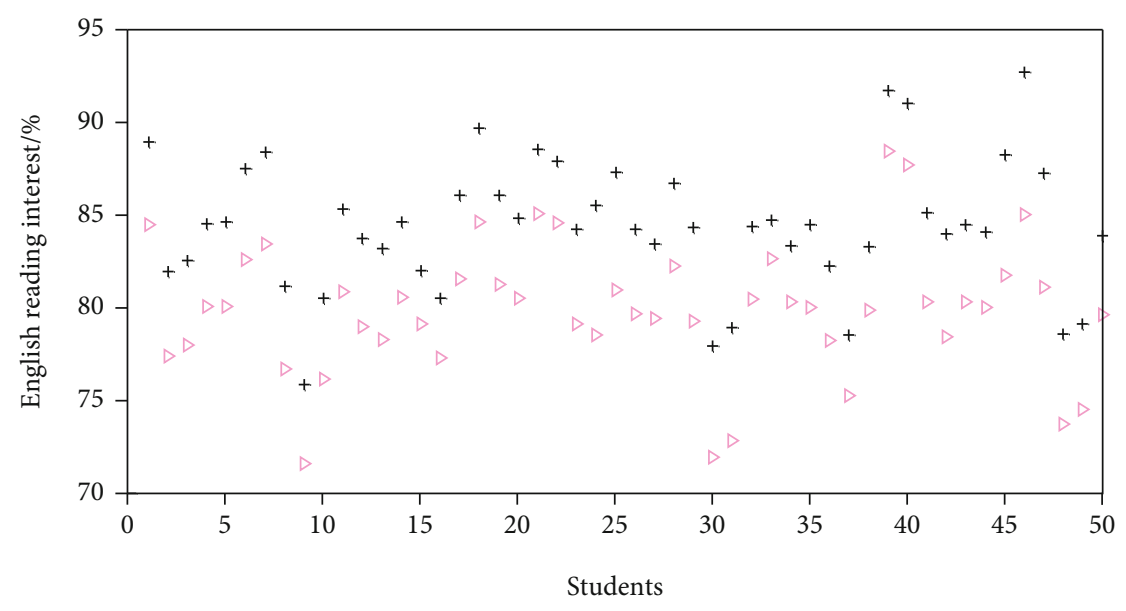

+50 students in the experimental class

$>50$ students in the control class

FIGURE 8: English reading interest in the dimension of affect and attitude.

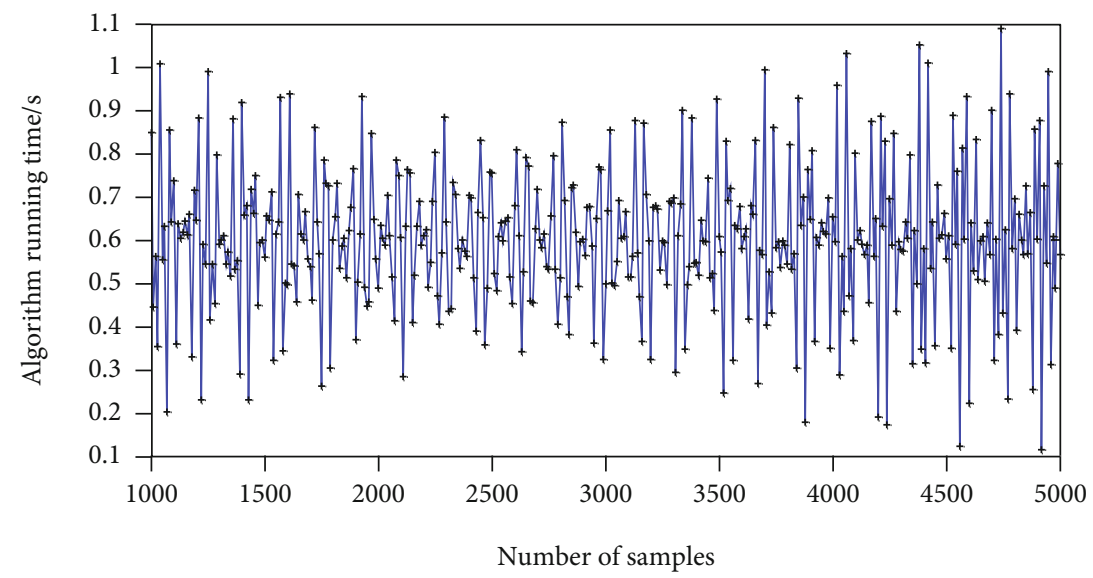

FIGURE 9: Running time of the global positioning algorithm for English reading text based on semantic segmentation. 
were distributed, 100 questionnaires were returned, and the recovery rate was $100 \%$. Among the 100 questionnaires returned, there was no invalid questionnaire. After sorting out the questionnaire score data, this paper uses SPSS22.0 to perform statistical analysis on these data. The results are shown in Figure 6.

\section{(1) Knowledge and skills}

The secondary dimensions of knowledge and skill dimensions include vocabulary, sentences, cultural background knowledge, and article information analysis. It can be seen from Table 2 that in terms of English knowledge and skills, learners' approval of the impact of English reading software on their English cultural background knowledge is the highest, followed by vocabulary, and the lowest is English article information analysis.

\section{(2) Process and method}

The process and method dimensions include the secondary dimensions of learning strategies and learning methods. During the experiment, the subjects used English reading software to practice English reading, and learners' learning strategies improved. In the process of experimental research, learners use English reading software to learn English during off-class time. Learners can study anytime, anywhere, or choose their favorite English articles for reading and recitation. During the period of experimental research, students changed and improved their English reading learning strategies, thereby improving their English reading ability. Figure 7 shows the proportion of approval degree of process and method dimensions. It can be seen that the proportion of people who strongly agree is the highest.

\section{(3) Emotional attitude}

The data in Figure 8 shows that learners have changed their learning interest in the process of using English reading software to learn English. By using English reading software, most learners prefer to read in English and also like to actively participate in learning activities.

5.4. Algorithm Running Time Test. The students in the experimental class used the semantic segmentation-based global positioning algorithm for English reading text proposed in this paper. In order to verify whether the algorithm can be applied to large-scale datasets, we conducted simulation experiments on the running time of the algorithm here. With a large-scale sample set, the running time of the global positioning algorithm for English reading text based on semantic segmentation is shown in Figure 9. It can be seen that the running time of the algorithm is less than $1.1 \mathrm{~s}$, which meets the real-time requirements.

\section{Conclusion}

We propose a segmented combined visual English text measurement method and build a complete English text measurement system based on this method. The system fully combines the advantages of the linear image sensor and the area image sensor in long-distance and short-distance English text measurement and can continuously perform high-precision English text tracking within a large range of distance changes. A dedicated image acquisition and processing circuit is designed for each line array camera of the remote measurement module. This circuit can flexibly acquire the target image and demodulate the imaging position of the target light source under the control of external signals. The design of the communication link combined with the system can ensure the imaging synchronization of the two sets of orthogonal line scan cameras on the hardware, which provides a guarantee for the realization of high-precision dynamic English text measurement. This paper analyzes the environment perception problems faced by the vehicle-mounted surround vision system in a complex environment and proposes the use of deep learning-based semantic segmentation technology for semantic segmentation, which provides a solid foundation for the global positioning of English reading text. The English word boundaries of the English text are extracted from the semantic segmentation image. Based on the plane assumption, they are projected to the coordinate system, combined with the English word boundary information marked in the highprecision image, and the two are matched to obtain the global initial positioning. With the rapid development of information technology, college English teaching is no longer a traditional classroom teaching, because it is impossible for students to acquire English knowledge only through school classroom reading teaching. Students need to learn English reading anytime and anywhere according to their own situation. The birth of reading software has provided great help for the further promotion of mobile language learning. Under the guidance of the concept of mobile learning, English reading teaching can be carried out in the form of integration of English reading software and English courses. Teachers should design an English classroom based on English reading software through a comprehensive analysis of English curriculum requirements and teaching content, so as to stimulate students' enthusiasm for learning and enhance their English language ability. In English reading learning, students should use mobile devices to promote their English learning based on the characteristics of English reading software. In addition, teachers can also guide students to use some good English reading software to learn English. English reading software not only makes it more convenient for students to learn English but also subtly improves students' autonomous learning ability.

\section{Data Availability}

The data used to support the findings of this study are available from the corresponding author upon request.

\section{Conflicts of Interest}

The authors declare that they have no known competing financial interests or personal relationships that could have appeared to influence the work reported in this paper. 


\section{References}

[1] H. Gu, J. Yao, P. Bai, L. Zhou, A. C. K. Cheung, and P. C. Abrami, "Does Abracadabra help improve the English reading ability of Chinese elementary school students? A quasi-natural experimental study," Science Insights Education Frontiers, vol. 9, no. 2, pp. 1221-1240, 2021.

[2] M. M. Hamad, "Using WhatsApp to enhance students' learning of English language" experience to share"," Higher Education Studies, vol. 7, no. 4, pp. 74-87, 2017.

[3] D. Hooshyar, M. Yousefi, and H. Lim, "A procedural content generation-based framework for educational games: toward a tailored data-driven game for developing early English reading skills," Journal of Educational Computing Research, vol. 56, no. 2, pp. 293-310, 2018.

[4] K. Nikolopoulou, D. Akriotou, and V. Gialamas, "Early reading skills in English as a foreign language via ICT in Greece: early childhood student teachers' perceptions," Early Childhood Education Journal, vol. 47, no. 5, pp. 597-606, 2019.

[5] Y. Chang, H. Zhou, X. Wang, L. Shen, and T. Hu, "Crossdrone binocular coordination for ground moving target tracking in occlusion-rich scenarios," IEEE Robotics and Automation Letters, vol. 5, no. 2, pp. 3161-3168, 2020.

[6] M. Zirnstein, J. G. van Hell, and J. F. Kroll, "Cognitive control ability mediates prediction costs in monolinguals and bilinguals," Cognition, vol. 176, pp. 87-106, 2018.

[7] M. Alyami and M. A. Mohsen, "The use of a reading lexicon to aid contextual vocabulary acquisition by EFL Arab learners," Journal of Psycholinguistic Research, vol. 48, no. 5, pp. 10051023, 2019.

[8] M. Song, "The impacts of extensive reading on EFL primary school students' vocabulary acquisition and reading comprehension," Journal of Extensive Reading, vol. 5, pp. 60-69, 2020.

[9] F. J. González-Castaño, F. Gil-Castineira, D. Rodriguez-Pereira, J. Á. Regueiro-Janeiro, S. Garcia-Mendez, and D. Candal-Ventureira, "Self-corrective sensor fusion for drone positioning in indoor facilities," IEEE Access, vol. 9, pp. 24152427, 2020.

[10] A. Kazemi, M. Bagheri, and E. Rassaei, "Impact of interventionist dynamic assessment on Iranian EFL learners' L2 reading comprehension and classroom engagement," International Journal of Foreign Language Teaching and Research, vol. 9, no. 39, pp. 67-79, 2021.

[11] F. Zhang, "Quality-improving strategies of college English teaching based on microlesson and flipped classroom," English Language Teaching, vol. 10, no. 5, pp. 243-249, 2017.

[12] M. M. T. Mohaidat, "The impact of electronic mind maps on students' reading comprehension," English Language Teaching, vol. 11, no. 4, pp. 32-42, 2018.

[13] R. Zhang, X. Jing, S. Wu, C. Jiang, J. Mu, and F. R. Yu, "Devicefree wireless sensing for human detection: the deep learning perspective," IEEE Internet of Things Journal, vol. 8, no. 4, pp. 2517-2539, 2021.

[14] S. Jozwik and A. L. Mustian, "Effects of technology-supported language experience approach for English learners with exceptional needs," Reading \& Writing Quarterly, vol. 36, no. 5, pp. 418-437, 2020.

[15] M. H. M. Jamrus and A. B. Razali, "Augmented reality in teaching and learning English reading: realities, possibilities, and limitations," International Journal of Academic Research in Progressive Education and Development, vol. 8, no. 4, pp. 724-737, 2019.
[16] X. Zeng, F. Zhang, B. Wang, and K. J. R. Liu, "Radio frequency based direction sensing using massive MIMO," IEEE Access, vol. 8, pp. 26827-26838, 2020.

[17] S. G. Ghalebandi and A. Noorhidawati, "Engaging children with pleasure reading: the e-reading experience," Journal of Educational Computing Research, vol. 56, no. 8, pp. 12131237, 2019.

[18] S. Radhika, R. D. Gomathi, and S. Nivedha, "Enhancing the reading comprehension of the tribal students using peculiar classroom methodology," Psychology and Education Journal, vol. 58, no. 2, pp. 5739-5743, 2021.

[19] J. Chen and W. Kim, "A human-following mobile robot providing natural and universal interfaces for control with wireless electronic devices," IEEE/ASME Transactions on Mechatronics, vol. 24, no. 5, pp. 2377-2385, 2019.

[20] D. L. Baker, D. L. Basaraba, K. Smolkowski et al., "Exploring the cross-linguistic transfer of reading skills in Spanish to English in the context of a computer adaptive reading intervention," Bilingual Research Journal, vol. 40, no. 2, pp. 222239, 2017.

[21] Z. Li, F. Nie, X. Chang, L. Nie, H. Zhang, and Y. Yang, "Rankconstrained spectral clustering with flexible embedding," IEEE transactions on neural networks and learning systems, vol. 29, no. 12, pp. 6073-6082, 2018.

[22] Z. Li, L. Yao, X. Chang, K. Zhan, J. Sun, and H. Zhang, "Zeroshot event detection via event-adaptive concept relevance mining," Pattern Recognition, vol. 88, pp. 595-603, 2019.

[23] Z. Li, F. Nie, X. Chang, Y. Yang, C. Zhang, and N. Sebe, "Dynamic affinity graph construction for spectral clustering using multiple features," IEEE Transactions on Neural Networks and Learning Systems, vol. 29, no. 12, pp. 6323-6332, 2018. 